\title{
Ultrasonic Method for Aircraft Wake Vortex Detection
}

\author{
Rebecca J. Rodenhiser ${ }^{*}$ William W. Durgin ${ }^{\dagger}$ and Hamid Johari ${ }^{\dagger}$ \\ Worcester Polytechnic Institute, Worcester, MA, 01609
}

\begin{abstract}
This paper describes the experimental proof of concept study for an ultrasonic method of wake vortex detection for airport use. This new acoustic method utilizes travel time of acoustic pulses around a closed path to measure the net circulation within the acoustic path. In this application the closed path encloses the vorticity shed from one side of a Piper PA-28 aircraft wing. Magnitude and sign of circulation detected is comparable to the expected circulation generated by the Piper PA-28 test aircraft. This study demonstrates the validity of the acoustic method in detecting aircraft wake vortices. Further investigations and applications utilizing this technique are discussed.
\end{abstract}

\section{Nomenclature}

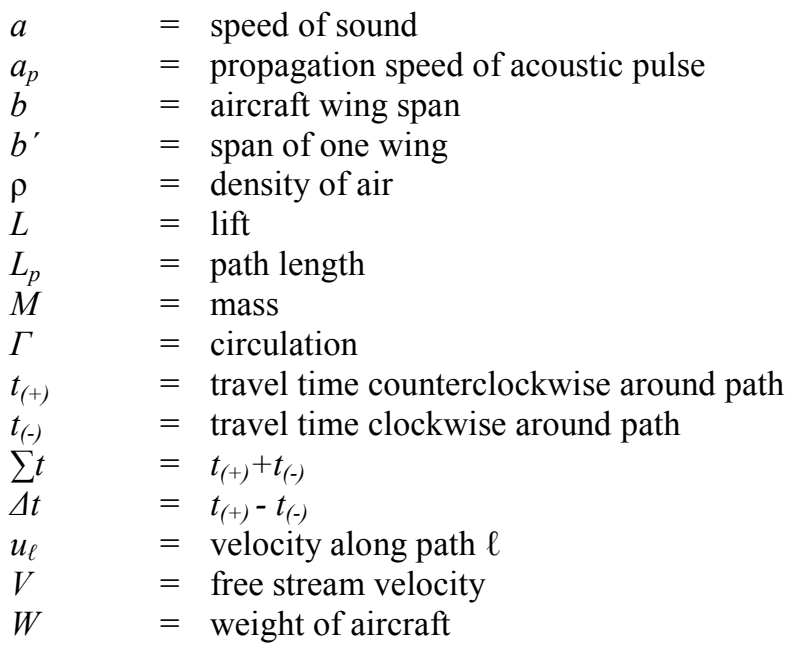

\section{Introduction}

$\mathrm{W}$ ake vortices pose a significant safety hazard to all air traffic and have been cited as the cause of many aircraft incidents and accidents. These vortices trail from the wings of any aircraft producing lift, and may contain intense velocities that can cause structural damage or rolling of any aircraft that encounter them. Detecting these wake vortices is highly desirable, particularly in the vicinity of airports where aircraft are in close proximity and share common flight paths making the probability of wake vortex encounters the greatest.

All lift-generating airfoils result in a bound circulation that occurs along the cross-section of the wing, the magnitude of which can be described by the Kutta-Joukowski theorem ${ }^{1}$. Knowing the initial load placed on the wing and the geometric measurements of the aircraft, the circulation can be theoretically calculated in the tip vortex $^{1-4}$. Numerous observational studies and numerical simulations have been conducted to better understand the motion and decay of these vortices. If unaffected by any outside forces, the vortices are observed to induce a downward motion on each other. The pair will descend in the atmosphere as they decay due to viscous forces, until they dissipate entirely or interact with an object or are affected by some outside force. ${ }^{5}$

*Graduate Student, Mechanical Engineering Department, 100 Institute Road, Worcester, MA 01609; Student Member AIAA

${ }^{\dagger}$ Professor, Mechanical Engineering Department, 100 Institute Road, Worcester, MA 01609; Associate Fellow AIAA 
Behavior of wake vortices is widely shown to be altered by proximity to the ground ${ }^{5-7}$ as well as by various atmospheric conditions. ${ }^{8,9}$ A numerical simulation by Corjon and Poinsot ${ }^{5}$ finds that ground proximity and resulting viscous flow produce a secondary pair of vortices that induce the original wake vortices to move upward; experimental studies confirm a formation of secondary vortices as a result of proximity to the ground. ${ }^{6}$ Proximity to the ground has also been shown in numerical simulations to produce lateral separation of the vortex pair. ${ }^{5,7}$

Real-time detection of wake vortices would result in the ability to create appropriate aircraft spacing based on actual duration of these vortices, instead of using the estimated values that are currently in place. As previous research shows, wake vortex dissipation can be enhanced by a variety of factors, and circulation may reduce to harmless levels well before the mandatory separation distance has been reached. Vortices can also be advected out of the flight path by local winds, leaving a safe runway available for air traffic. In a 2000 study it is revealed that the financial implications of reducing the current separation distances by an average of 30 seconds would result in net savings of five billion US dollars annually for the airline industry. ${ }^{10}$

Detection of wake vortices at airports has been attempted by numerous methods. Attempts have been made to observe actual wake vortices using radar, ${ }^{11}$ Lidar, ${ }^{12}$ and a radio acoustic sounding system (RASS). ${ }^{10,13}$ The National Aeronautics and Space Administration (NASA) is working toward a comprehensive wake vortex detection method that incorporates local atmospheric conditions, actual circulation data, and numerical modeling to produce a wake vortex strength and location prediction tool. ${ }^{12,14,15}$ This NASA project title AVOSS - Aircraft VOrtex Spacing System - has conducted field trials at Dallas/ Fort Worth Airport. Another technology known as SOCRATES has been utilized in field studies to track and measure the presence of wake vortices. SOCRATES (Sensor for Optically Characterizing Remote Atmospheric Turbulence Emanating Sound) is a technology that measures the infrasound emitted from the vortex structures in the $200-300 \mathrm{~Hz}$ frequency range. ${ }^{16}$ These methods have been field tested with varying degrees of success, yet no method has yet resulted in a system capable of vortex detection for reduction in aircraft spacing standards.

In this study, a new ultrasonic method of vortex detection was deployed at an airport to detect wake vortex circulations from aircraft during low pass flights over the airfield. This method of wake vortex detection has been utilized previously in a wind tunnel to accurately measure the circulation of a wing tip vortex behind a NACA 0012 airfoil. ${ }^{1}$ This current study is the first attempt to test the viability of this methodology in a realistic airport environment. This technology is able to detect wake circulations that are in ground effect, an ability lacking in many other detection systems.

\section{Methodology}

This method of circulation detection involves using acoustic pulses to determine the velocity component of a fluid along a closed path, and from that determine the circulation within that path. The use of acoustics in circulation detection was first achieved by Schmidt. ${ }^{17}$ The technique was first applied utilizing a closed path to detect the trailing circulation from a wing in wind tunnel experiments. ${ }^{18}$ This method of circulation detection using a

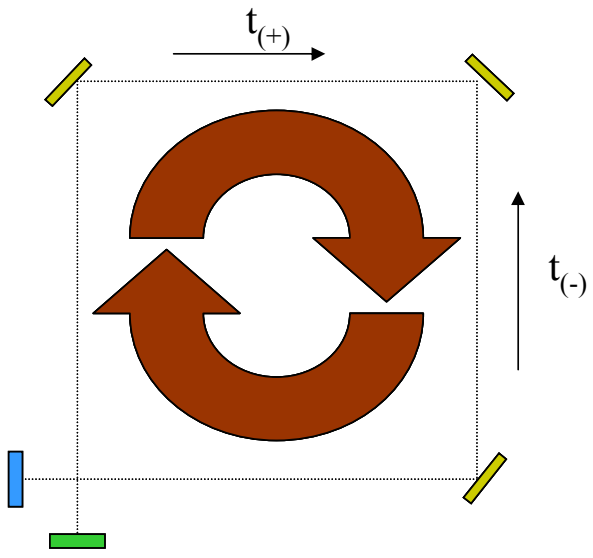

Figure 1 - Schematic of acoustic circulation measurement technique. closed path, ${ }^{3}$ involves transmitting a focused acoustic signal along the closed path around a cross section of circulation. The acoustic signal travel time around the path is used to measure the line integral of velocity along the acoustic path. The signal is sent around the closed path in clockwise and counterclockwise directions, and the travel time difference between the opposite-traveling pulses is shown to be directly correlated to the circulation contained within the path, as depicted in Fig. 1.

With proper positioning of our closed path to capture the wake vortex of an aircraft, measuring the travel time of the signal along the path will result in determination of net wake vortex circulation enclosed by the path. Development of this technique is reviewed below; complete discussion and analysis of this technique can be found in previous literature. ${ }^{19}$

The basic principle used in this calculation is the definition of circulation contained within a path is the line integral of velocity along that path, shown in Eq. (1). This inline velocity along the pulse path is measured through timing the ultrasonic pulse traveling that path. The travel 
time of the acoustic pulse is the integral of the inverse propagation speed of sound in that fluid, shown in Eq. (2). The propagation speed is simply the speed of sound plus the velocity along the path.

$$
\begin{gathered}
\Gamma=\oint \vec{u}_{l} \cdot d \vec{\ell}=\int_{c_{\text {closed_path }} u_{\ell} d \ell} \\
t=\int \frac{1}{a_{p}} d \ell
\end{gathered}
$$

The travel time of the pulse along the path is measured for travel in both clockwise and counterclockwise directions, resulting in two travel time values, denoted as $t_{(+)}$and $t_{(-)}$. Expanding Eq. (2) for each direction of travel, we arrive at Eqs. (3) and (4). The two travel times in Eqs. (3) and (4) area shown schematically in Fig. 1.

$$
\begin{aligned}
& t_{(+)}=\int \frac{1}{a+u_{\ell}} d \ell \\
& t_{(-)}=\int \frac{1}{a-u_{\ell}} d \ell
\end{aligned}
$$

Finding the difference in the propagation time for these pulses results in Eq. (5). Assuming that the local fluid velocity is much smaller than the speed of sound, in other words $\left(u_{\ell} / a\right)^{2}<<1$, Eq. (5) can be reduced into the form shown in Eq. (6).

$$
\begin{gathered}
\Delta t=t_{(-)}-t_{(+)}=\frac{2}{\bar{a}^{2}} \int \frac{u_{\ell}}{1-\left(u_{\ell} / a\right)^{2}} \\
\Delta t=\frac{2}{\bar{a}^{2}} \int u_{\ell} \partial \ell
\end{gathered}
$$

Our pulse travel time measurement can again be used to calculate the average speed of sound shown in Eq. (7) as twice the path length divided by the sum of the pulse transmission in opposite directions. Eq. (7) along with the definition of circulation, found in Eq. (1), is then substituted into Eq. (6), and is shown below in Eq. (8).

$$
\bar{a}=\frac{2 L_{p}}{t_{(+)}+t_{(-)}}
$$




$$
\Gamma=2(\Delta t)\left[\frac{L_{p}}{\sum t}\right]^{2}
$$

It is observed from Eq. (8) that the circulation contained within the closed path can be calculated as a function of the path length and propagation times of acoustic pulses traveling the path in opposite directions. The only variable in the equation is the propagation times of the acoustic pulses (as a path length will be a constant for the setup), and therefore is the only quantity that needs to be measured to calculate circulation. Advantages to this method include no need for equipment calibration, and the elimination of the speed of sound from the equation. Fluctuations in speed of sound over time will occur, and are accounted for in the equation, making it unnecessary to track fluctuations in the variables that affect speed of sound such as temperature, humidity, and pressure.

\section{Experimental Design}

The theory of acoustic circulation detection described in the previous section had to be applied to a very large scale in order to measure actual aircraft trailing vortices. Acoustic frequency was lowered from previous wind tunnel experiments to allow for longer transmission distances, and specialty equipment was located that would allow for focused acoustic transmission over great distances. A schematic of the equipment utilized in the prototype $\mathrm{s}$ ystem developed for airport use is shown in Figure 2.

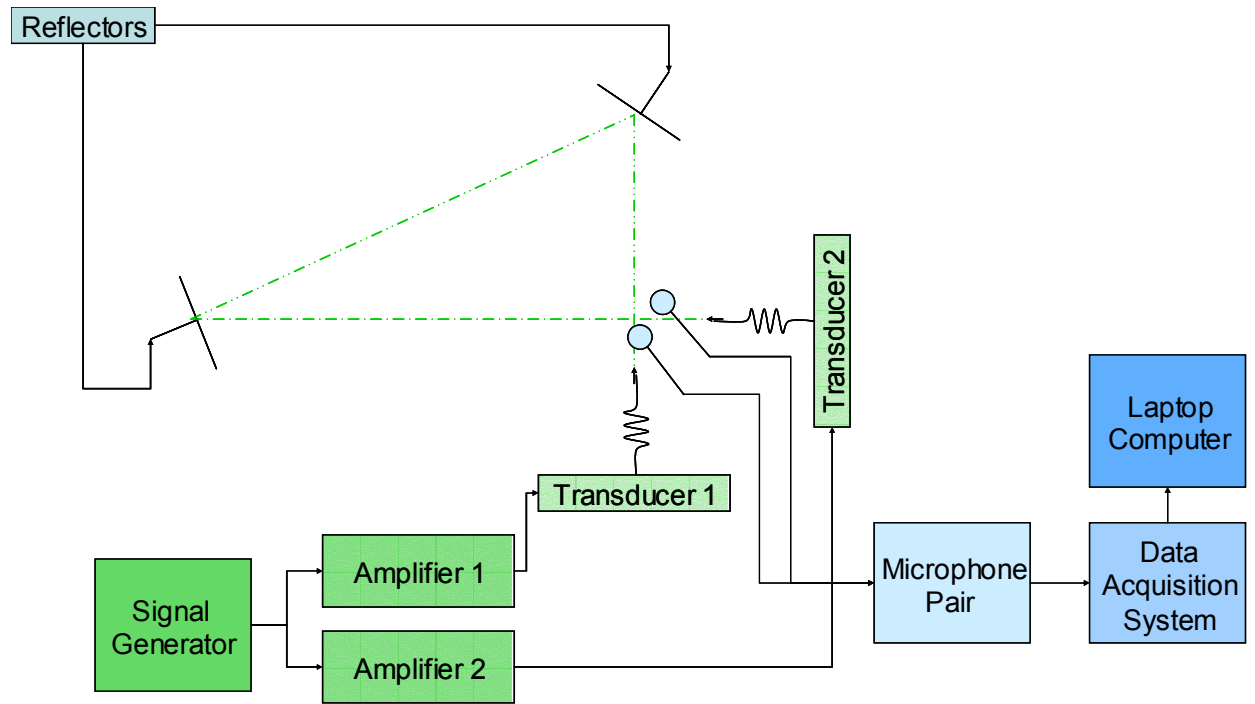

Figure 2 - Equipment used in prototype system.

The closed path used in this study is triangular in shape, and was accomplished by mounting one reflector atop a 30 foot tower, and the other approximately 80 feet away at ground height, as shown in Fig. 3. The signals originated from two transducers at the bottom of the tower, positioned to transmit the ultrasonic signal in opposite directions, and transmitting in an alternating fashion. A 5-cycle sine wave packet, at a frequency of $57 \mathrm{kHz}$ and output power of 130 decibels is transmitted around the path, alternating in direction, and detected at the beginning and end of the path by microphones at the base of the tower, see Fig. 4. The test plane was flown approximately 15 feet above ground and positioned such that one wingtip went within the closed path, and the other outside the region. The goal was to capture a single vortex within the path, yielding detection of the maximum amount of circulation. 


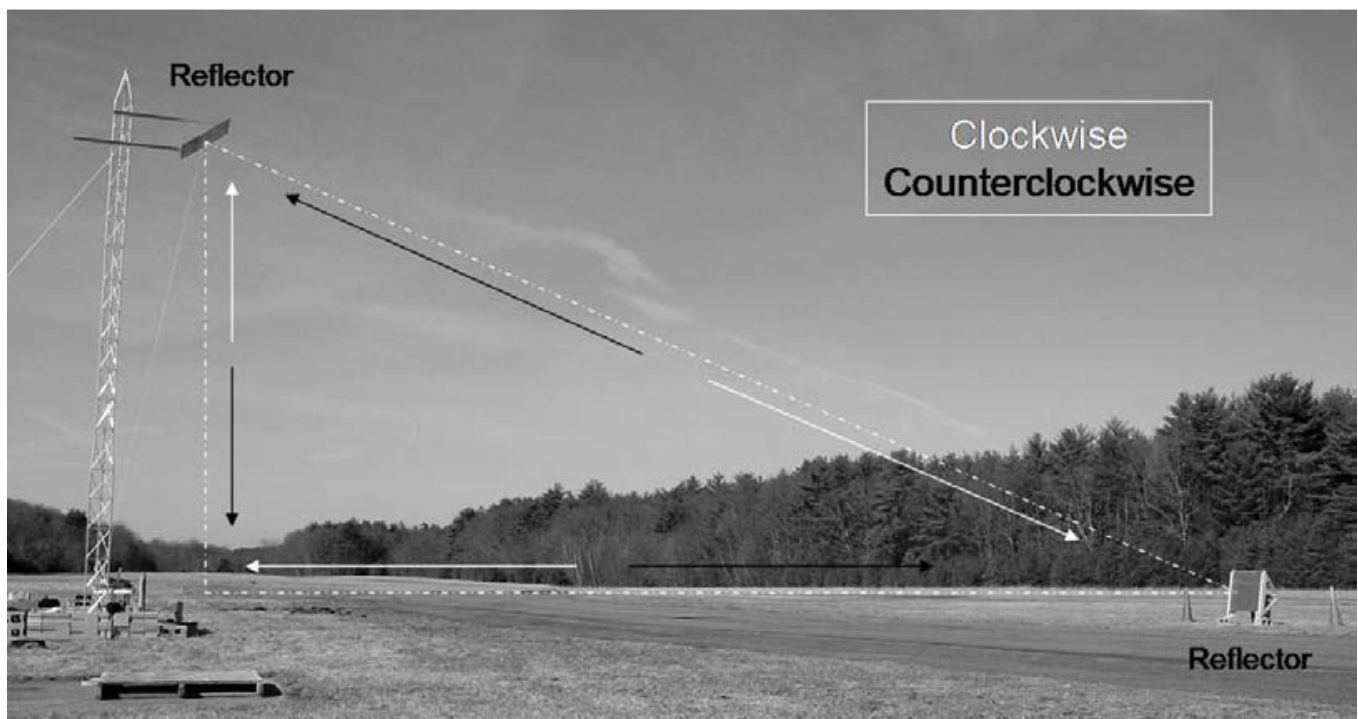

Figure 3. Test Equipment Setup Showing Directions of Signal Transmission

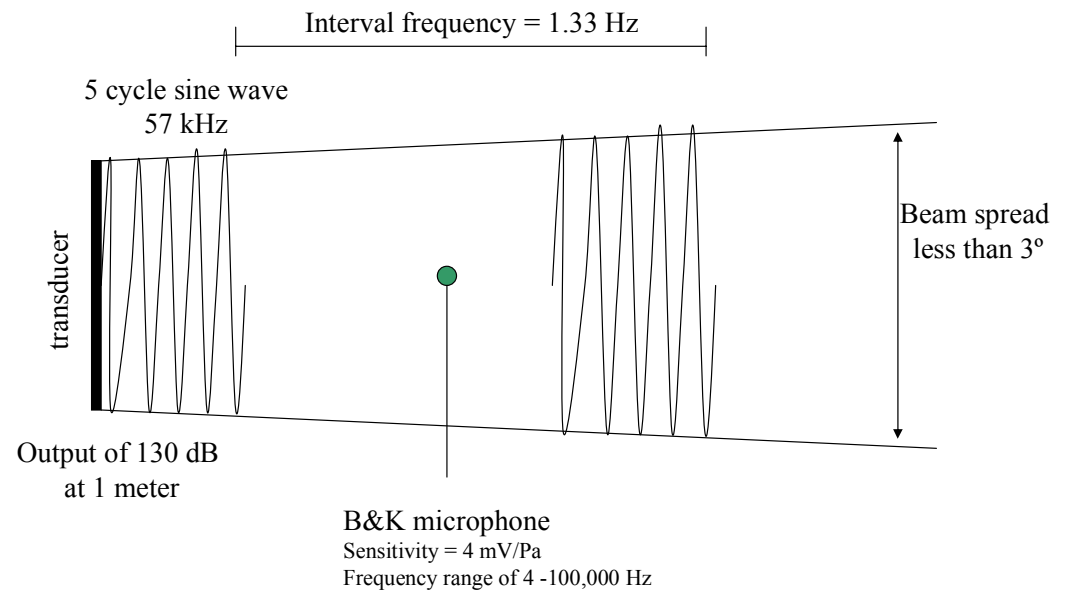

Figure 4 - Acoustic transducer and microphone details.

The ultrasonic signal is monitored using microphones and collected through a data acquisition system sampling at $285 \mathrm{kHz}$, controlled by a laptop computer using Lab View Software. The signals detected at the beginning and end of the path are filtered using a high-pass Butterworth filter, amplitudes are normalized, and signals are correlated using a cross-correlation function. A Hilbert transform envelope function was then applied to the correlation function, the maximum of which was used to calculate the travel time of the pulse. Schematics of the signal processing are shown in Fig. 5.

The travel times were then compared for successive pulses to determine the difference in travel time. These time values were converted to circulation using Eq. (8). The interval of pulse transmission was 0.75 seconds, resulting in a sampling frequency of $1.33 \mathrm{~Hz}$ over an approximately 2 minute sampling period. An attempt was made to capture 30 seconds of data prior to aircraft fly-by for each trial.

There is an inherent error present within this measuring system due to sampling and correlation technique. The data acquisition system introduces an error of magnitude $1 /($ sample rate), which is $1 /(285000 \mathrm{~Hz})=3.5 \mu$ s. The cross correlation technique should yield an error no greater than one cycle, which is $1 /(57000 \mathrm{~Hz})=18$ microseconds. The peak detection technique using the Hilbert Envelope function should introduce an error no greater than one-half a cycle, or 9 microseconds. The total error from all three sources can be determined using a standard error in sum formula, which is calculated to be $20 \mu \mathrm{s}$. 

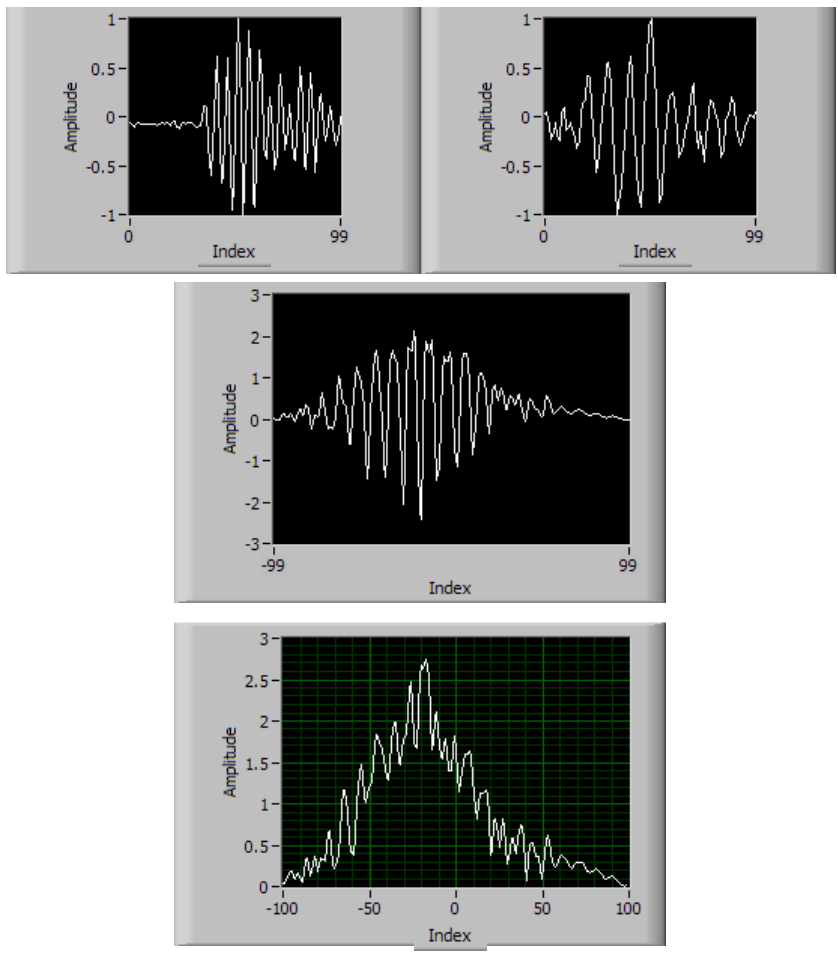

Figure 5 - Signal processing: Transmitted and received signals, correlation function, and Hilbert envelope function.

\section{Results and Discussion}

Testing was conducted during two days at Tanner-Hiller Airport, located west of Worcester, Massachusetts. The aircraft used was a four seat Piper (PA-28) aircraft, with rectangular wings spanning 9.1 meters. Multiple tests were done on each day, with slight variations in flight path. Testing dates were chosen based on meteorological conditions; testing was performed in the early morning hours when temperatures were cool and winds were calm. An attempt was made to complete testing for the day before any daytime convection began, which for the most part was achieved.

An expected circulation value of the wake vortex generated by the aircraft was estimated for each trial flight. Knowledge of the aircraft weight, flight speed during fly-by, wing span and density of air, enable a calculation of the theoretical vortex strength shed behind each wing using the Kutta-Joukowski theorem ${ }^{1}$ shown in Eq. (9). Table 1 shows a summary of the meteorological conditions and aircraft statistics for each trial conducted. Flap settings range from 0-3, indicating no flaps to full flaps, respectively. Lift was approximated by the mass of the aircraft and contents multiplied by gravity since level flight was maintained while aircraft passed test area. Air density was assumed to be $1.25 \mathrm{~kg} / \mathrm{m}^{3}$, and wing semi-span $\mathrm{b}^{\prime}$ is 4 meters. It is expected that the circulation will be positive based on the direction of flight and method of $\Delta t$ calculations.

$$
\Gamma \approx \frac{(1 / 2) L}{\rho \mathrm{Vb}^{\prime}} \approx \frac{M g}{2 \rho V \mathrm{~b}^{\prime}}
$$


Table 1. Meteorological and Aircraft Information for each Trial Flight.

\begin{tabular}{|c|c|c|c|c|c|c|c|c|}
\hline Trial & Date & $\begin{array}{c}\text { Temp } \\
\left({ }^{\circ} \mathrm{C}\right)\end{array}$ & $\begin{array}{c}\text { Winds } \\
(\text { observed })\end{array}$ & $\begin{array}{c}\text { Flight } \\
\text { Path }\end{array}$ & $\begin{array}{c}\text { Aircraft } \\
\text { Mass }\end{array}$ & $\begin{array}{c}\text { Flight } \\
\text { Speed }\end{array}$ & $\begin{array}{c}\text { Flap } \\
\text { Setting }\end{array}$ & $\begin{array}{c}\text { Circulation } \\
\left(\mathrm{m}^{2} / \mathrm{s}\right)\end{array}$ \\
\hline 1 & Day 1 & 7 & Calm & A & $993 \mathrm{~kg}$ & $31 \mathrm{~m} / \mathrm{s}$ & 3 & 31.4 \\
\hline 2 & Day 1 & 7 & Calm & A & $993 \mathrm{~kg}$ & $30 \mathrm{~m} / \mathrm{s}$ & 3 & 32.4 \\
\hline 3 & Day 1 & 7 & Calm & A & $993 \mathrm{~kg}$ & $31 \mathrm{~m} / \mathrm{s}$ & 3 & 31.4 \\
\hline 4 & Day 1 & 8 & Calm & A & $993 \mathrm{~kg}$ & $31 \mathrm{~m} / \mathrm{s}$ & 3 & 31.4 \\
\hline 5 & Day 1 & 8 & Slight breeze & A & $993 \mathrm{~kg}$ & $31 \mathrm{~m} / \mathrm{s}$ & 3 & 31.4 \\
\hline 6 & Day 2 & -1 & Calm & A & $1012 \mathrm{~kg}$ & $36 \mathrm{~m} / \mathrm{s}$ & 3 & 27.6 \\
\hline 7 & Day 2 & -1 & Calm & A & $1012 \mathrm{~kg}$ & $33 \mathrm{~m} / \mathrm{s}$ & 3 & 30.1 \\
\hline 8 & Day 2 & -1 & Calm & A & $1012 \mathrm{~kg}$ & $35 \mathrm{~m} / \mathrm{s}$ & 3 & 28.3 \\
\hline 9 & Day 2 & 0 & Calm & C & $1012 \mathrm{~kg}$ & $35 \mathrm{~m} / \mathrm{s}$ & 3 & 28.3 \\
\hline 10 & Day 2 & 0 & Calm & C & $1012 \mathrm{~kg}$ & $35 \mathrm{~m} / \mathrm{s}$ & 3 & 28.3 \\
\hline 11 & Day 2 & 0 & Slight breeze & C & $1012 \mathrm{~kg}$ & $34 \mathrm{~m} / \mathrm{s}$ & 2 & 29.2 \\
\hline 12 & Day 2 & 3 & Slight breeze & B & $1012 \mathrm{~kg}$ & $30 \mathrm{~m} / \mathrm{s}$ & 3 & 33.1 \\
\hline 13 & Day 2 & 3 & Slight breeze & B & $1012 \mathrm{~kg}$ & $32 \mathrm{~m} / \mathrm{s}$ & 2 & 31.0 \\
\hline 14 & Day 2 & 3 & Slight breeze & B & $1012 \mathrm{~kg}$ & $32 \mathrm{~m} / \mathrm{s}$ & 2 & 31.0 \\
\hline
\end{tabular}

Figure 6 shows data from one trial run, with the aircraft passing the measurement equipment at time zero, and circulation is plotted in points with a five point running average shown with the blue curve. It can be seen from this plot that circulation data present prior to the aircraft arrival is essentially zero. After the aircraft passes, there is an immediate spike in circulation, which decays over approximately 45 seconds to pre-aircraft levels.

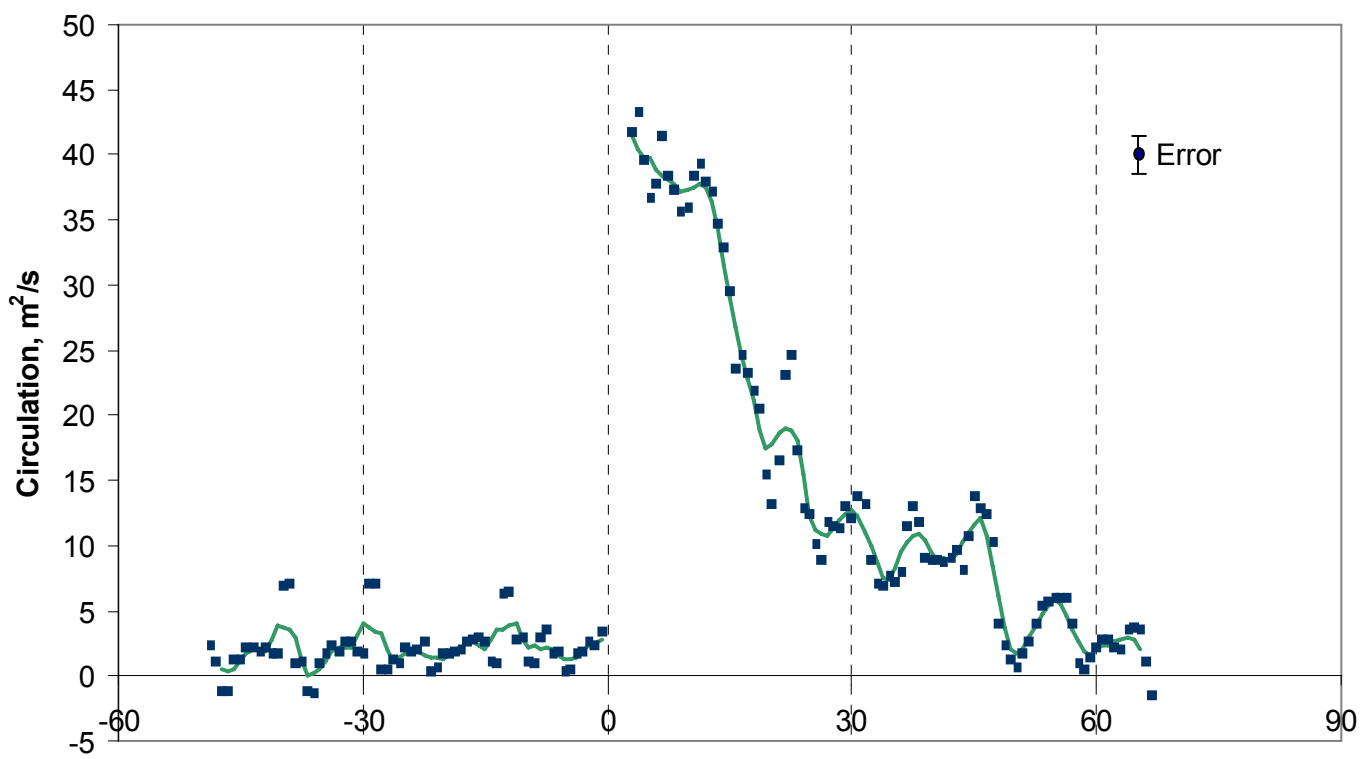

Time, $\mathbf{s}$

Figure 6. Actual data and running average for Trial 6

Despite pilot attempts to place the aircraft precisely in the designated path, variations of flight path did occur. During certain flights the plane was witnessed to be shifted horizontally or vertically by several feet of ideal placement. Variations in aircraft placement will result in variations in the duration the vortex remains within our measurement area, and therefore variations in the decay rate may be observed. Figure 7 shows multiple trials plotted onto a single graph. The overall consistency is observed in the shape of the circulation over time, yet slight variations are observed between flights due to the variability in flight path described above. 


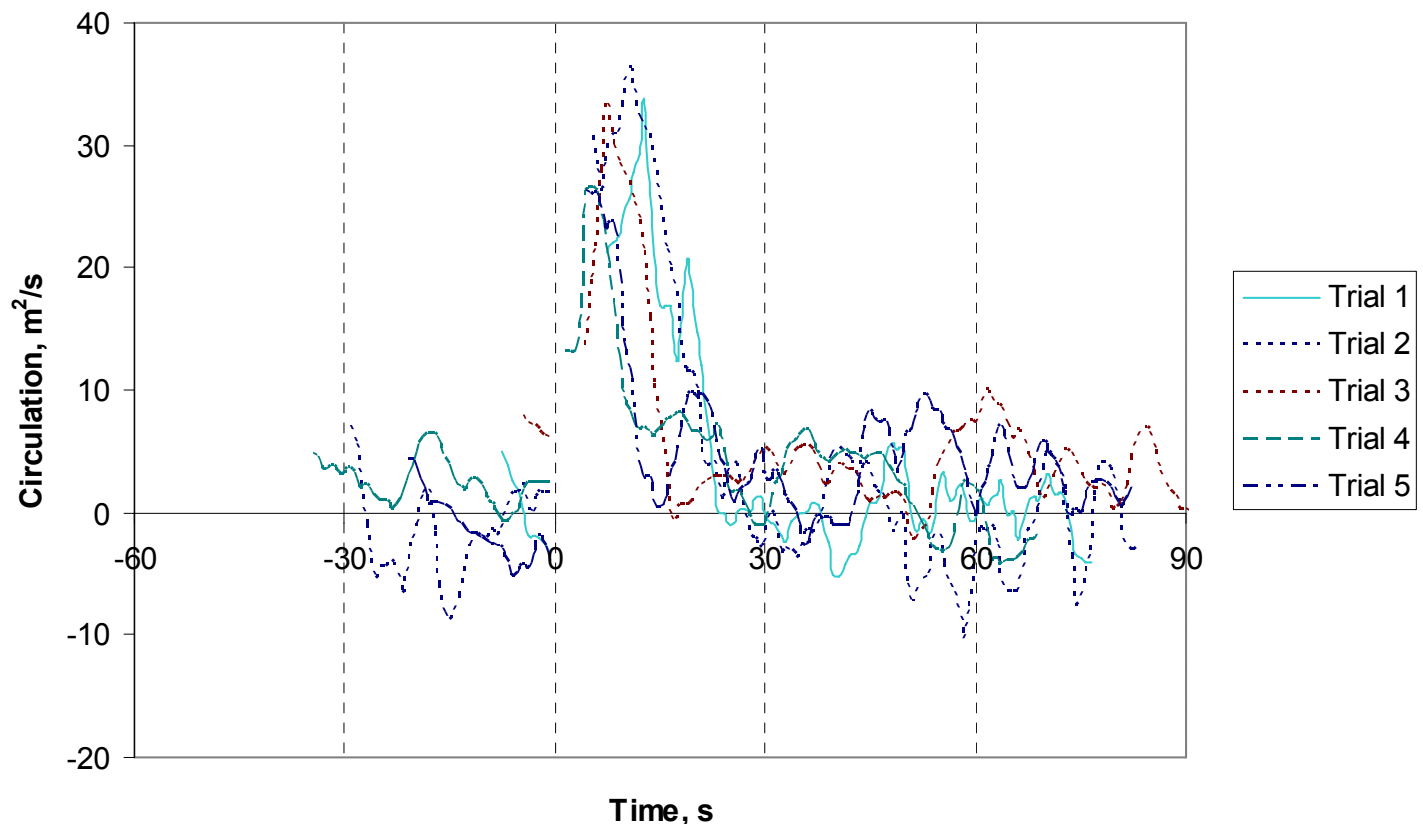

Figure 7 - Circulation vs. time history for trials 1-5.

These trials shown in Figs. 6 and 7 were carried out in conditions of little or no wind, and no atmospheric convection. These times were specifically chosen to minimize the amount of ambient atmospheric circulation present, which would be detected by the system. However, if there is a cross-wind present or ambient convection, vorticity is generated within the planetary boundary layer, and this will be measured by the equipment. Additional testing was conducted with no aircraft, to determine the atmospheric circulation that can be observed simply due to cross winds and convection.

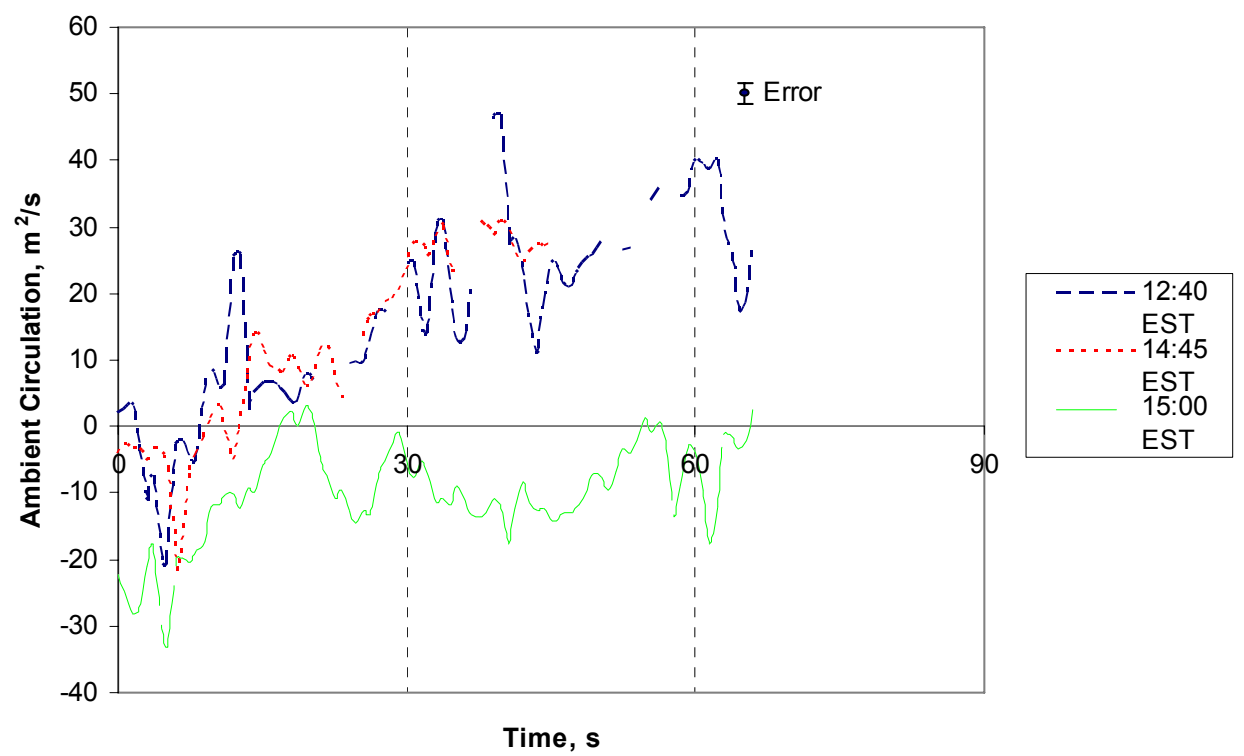

Figure 8 - Ambient circulation measurements due to cross wind. 
Our measurements are conducted primarily in the surface layer of the planetary boundary layer, which is defined by wind shear increasing logarithmically with height. Given that the chosen area shown in Fig. 3 varies with height (a greater portion of the triangular shaped area is closer to the surface than at the top of the tower), it is important to understand the distribution of vorticity as a function of height within the surface layer. Data were collected during times of cross-wind, with no airplane traffic. The goal was to measure the ambient circulation produced by crosswinds in the atmosphere. Fig. 8 displays ambient circulation data in measurement area when winds measured at surrounding National Weather Service observing station to be between 5 and 10 knots for the afternoon.

Knowledge of the vorticity gradient with height and our defined measurement area, we can calculate the ambient circulation due to the wind shear of the PBL. However, knowing that wind speed varies logarithmic with height is not of general practical use because that is an averaged, idealized wind shear relationship. In actuality, there are turbulent eddies present in the PBL, which disrupts the typical wind distribution with height. In addition, surface heating generates convective plumes and turbulent eddies, which also change the structure of the wind field in the surface layer. ${ }^{20,21}$ Attempting to discriminate the fraction of circulation observed by the acoustic system that results of a trailing vortex or PBL wind shear at a given instant in time can prove very difficult.

While calculating an exact instantaneous measurement of PBL circulation for a specific area is nearly impossible, it is believed that a reasonable estimate could be obtained. By placing an anemometer at the top of the tower, we could observe the wind direction and speed, and calculate the component of the wind that is along our measurement area.

An estimate of the ambient circulation present during the cross wind trials is shown in Fig. 8. Lowest wind speeds were observed to be 5 knots, which corresponds to approximately $2.5 \mathrm{~m} / \mathrm{s}$. As a first estimate, we can assume the vertical wind shear is constant within the 9 meter height of the acoustic path. Assuming constant wind shear and no vertical wind flow, vorticity is simplified, Eq. 10, with horizontal and vertical directions represented with $\mathrm{x}$ and $\mathrm{y}$, respectively. If the horizontal velocity gradient within the path can be ignored, a constant vorticity is present within the path.

$$
\omega \equiv \frac{\partial v}{\partial x}-\frac{\partial u}{\partial y} \approx \frac{\partial u}{\partial y}
$$

This yields a constant vorticity value for the entire measurement area of $0.28 \mathrm{~s}^{-1}$. Our measurement area is approximately $90 \mathrm{~m}^{2}$, which results in an ambient circulation value of $25.2 \mathrm{~m}^{2} / \mathrm{s}$. Similarly, with the highest wind speeds observed at $10 \mathrm{knots}$, or $5 \mathrm{~m} / \mathrm{s}$, the resulting ambient circulation, based on a linear shear profile with height, would be $50 \mathrm{~m}^{2} / \mathrm{s}$. Therefore, using these estimates, we would expect ambient circulation to vary between 25 and 50 $\mathrm{m}^{2} / \mathrm{s}$ assuming wind speeds were maintained between 5 and 10 knots.

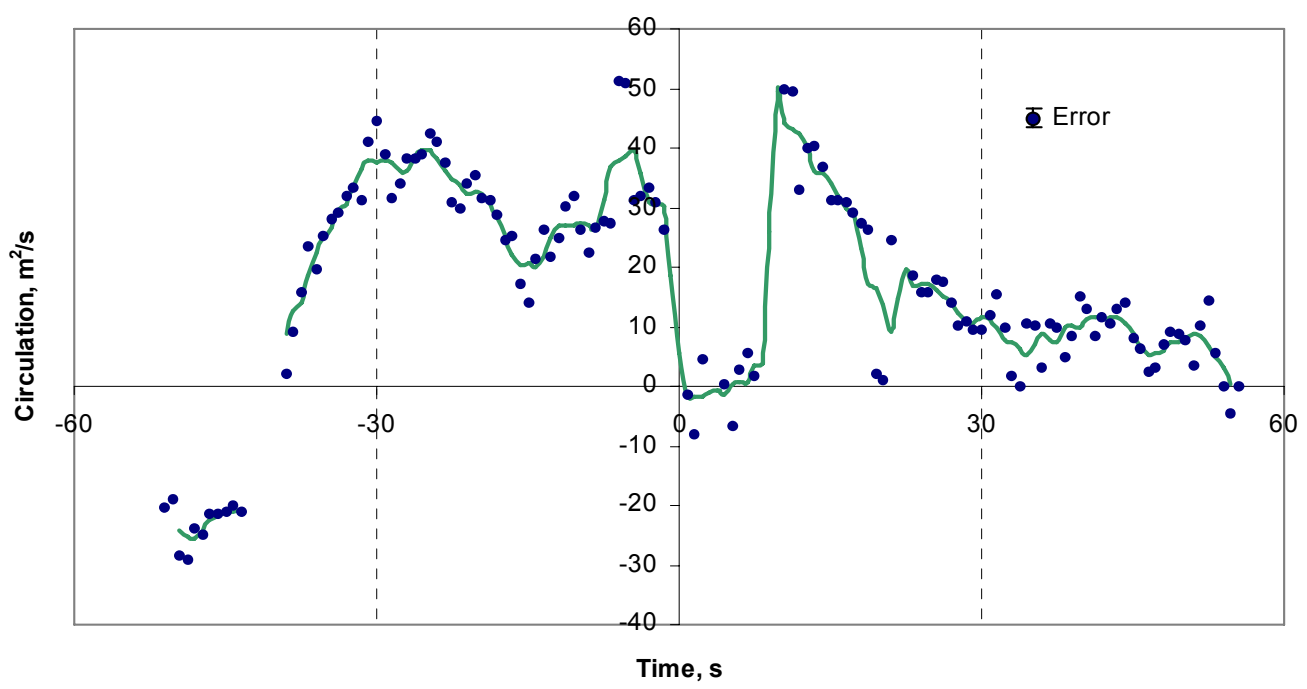

Figure 9 - Actual data and running average for trial with cross-wind. 
It is expected that the vortices generated by a small aircraft, such as the four-seat Piper used in this study, would not be clearly detectable in a cross-wind of 5 to 10 knots. The ambient circulations in our defined measurement area in such a scenario are equivalent to the intensity of the generated vortices. Measurements were attempted in this cross-wind environment with the Piper aircraft, with the results of one flight shown in Fig. 9. The aircraft flight path used for this trial would result in an expected negative value of circulation immediately after the aircrafts passage. It is observed that there is a negative trend in the data immediately after aircraft passage, although the wake vortex cannot definitively be discerned from the ambient circulation levels. While Fig. 9 supports the expectation that small vortices are not discernable in a moderate cross-wind of 5 to 10 knots, large commercial aircraft are observed to have vortices with initial strength of $300-500 \mathrm{~m}^{2} / \mathrm{s}^{22}$, and therefore would be easily detected in cross-wind.

Having instantaneous wind data from an anemometer at the top of the tower would provide us with a method for accounting for some of these ambient circulations, and possibly discriminating between these circulations and those produced by a wake vortex. Of course, turbulent fluctuations will ensure the vorticity profile with height is never constantly uniform, however initial calculations show it can provide a good estimate of in-situ calculations. With a responsive anemometer that provides data at the same time resolution as our measurement system $(1.33 \mathrm{~Hz})$, we can observe temporal changes in ambient circulations simultaneously with circulation measurements. When considering measuring vortices from large aircraft with initial strengths of $300-500 \mathrm{~m}^{2} / \mathrm{s}$ (Sarpkaya, 1998), the ambient circulation fluctuations of $25-50 \mathrm{~m}^{2} / \mathrm{s}$ at most amount to $10 \%$.

\section{Conclusions}

This study has shown that ultrasonic detection of circulation is a viable means for wake vortex detection in an airport environment. Vortices can be detected in the wake of small aircraft, proving this apparatus is sensitive enough to capture the smallest wake vortices. Vortex direction and intensity were clearly determined during multiple low passes of test aircraft. Margin of error of the measurement system remains well below the value of circulation shed from the smallest aircraft, and it is expected to remain negligible for larger circulation values from larger aircraft.

The system operates at its best in conditions of low wind with little ambient atmospheric circulation; these are also the environmental conditions when wake vortices can pose the largest hazard by remaining stationary on a runway. In calm conditions, vortex signatures are clearly visible, and results from multiple trials show very consistent results. In conditions of ambient winds, specifically a cross wind, natural variations of circulation can be an order of magnitude greater than in conditions of no wind. These ambient circulation values for a 5-10 knot cross wind are shown to be an order of magnitude less than wake vortices produced by heavy aircraft.

In summary, the current field trials support the following conclusions:

1) Measurement system is able to detect the magnitude and direction of aircraft wake vortex circulations

2) Multiple trials reveal similar vortex circulation time histories

3) Cross winds generate circulation within the planetary boundary layer that is detected by the system. Calculations show these circulations are an order of magnitude less than dangerous vortices produced by heavy aircraft.

Further studies will work to produce a real-time display of circulation data in the field. Additional configurations will also provide for the towers to be significantly spaced from the runway, so as not to pose an additional hazard to air traffic. Future work will also aim to increase the distance of the path length through customized equipment and adaptations to current path design. Performance in various meteorological conditions will be examined as well.

\section{References}

${ }^{1}$ Anderson, J., Fundamentals of Aerodynamics, McGraw-Hill, New York, 1984, Chaps. 3-6.

${ }^{2}$ Brown, C.E., "Aerodynamics of Wake Vortices,” AIAA Journal, Vol. 11, 1973, pp. 31-536.

${ }^{3}$ Gerz, T., and Holfzapel, F., "Wing-Tip Vortices, Turbulence, and the Distribution of Emissions," AIAA Journal, Vol. 37 , 1999, pp. 1270-1276.

${ }^{4}$ Ozger, E., Schell, I., and Jacob, D., "On the Structure and Attenuation of an Aircraft Wake," Journal of Aircraft, Vol. 38, 2001, pp. 878-887.

${ }^{5}$ Corjon, A., and Poinsot, T., "Wake Vortices Behavior Near Ground,” ESAIM: Proceedings, Vol. 1, 1996, pp. $279-294$.

${ }^{6}$ Burnham, D., and Hallock, J., "Measurements of Wake Vortices Interacting With the Ground," Journal of Aircraft, Vol. 42, 2005, pp. 1179-1187.

${ }^{7}$ Hamilton, D., and Proctor, F., "Wake Vortex Transport in Proximity to the Ground," $16^{\text {th }}$ Digital Avionics Systems Conference, 2000

${ }^{8}$ Holfzapel, F., Gerz, T., Fresch, M., and Dornbrack, A., "Wake Vortices in Convective Boundary Layer and Their Influence on Following Aircraft," Journal of Aircraft, Vol. 37, 2000, pp. 1001-1007. 
${ }^{9}$ Fresch, M., and Zinner, T., “Concept of Wake Vortex Behavior Class,” Journal of Aircraft, Vol. 41, 2004, pp. 564-570.

${ }^{10}$ Rubin, W., Burnham, D., Spitzer, E., and Rudis, R., "Robust Low Cost Airport Wake Vortex Sensor," Journal of Aircraft, Vol. 37, 2000, pp. 377-382.

${ }^{11}$ Shariff, K., and Wray, A., "Analysis of the Radar Reflectivity of Aircraft Vortex Wakes,” Journal of Fluid Mechanics, Vol. 463, 2002, pp. 121-161.

${ }^{12}$ Hinton, D., et. al., "NASA Aircraft Vortex Spacing System Development Status," AIAA 99-0753, 37 $7^{\text {th }}$ Aerospace Sciences Meeting, 1999.

${ }^{13}$ Boluriaan, S. and Morris, P., "Numerical Simulation of Wake Vortex Detection Using a Radio Acoustic Sounding System," AIAA Journal, Vol. 39, 2001, pp. 1097-1105.

${ }^{14}$ Hinton, D., "Description of Selected Algorithms and Implementation Details of a Concept-Demonstration Aircraft Vortex Spacing System," NASA TM-211027, 2001.

${ }^{15}$ O'Conner, C., and Rutishauser, D., "Enhanced Airport Capacity Through Safe, Dynamic Reductions in Aircraft Separation: NASA's Aircraft Vortex Spacing System," NASA TM-211052, 2001.

${ }^{16}$ Flight Safety Technologies, "Socrates Wake Vortex Detection and Tracking: A Status Report," November 2004.

${ }^{17}$ Schmidt, D., "Acoustical Method for Fast Detection and Measurement of Vortices in Wind Tunnels," ICIASF Record, 1975, pp. 216-228.

${ }^{18}$ Desabrais, K., and Johari, H., "Direct Circulation Measurement of a Tip Vortex," AIAA Journal, Vol. 38, 2000, pp. 21892191.

${ }^{19}$ Johari, H., and Durgin, W., "Direct Measurement of Circulation Using Ultrasound," Experiments in Fluids, Vol. 25, 1998, pp. 445-454.

${ }^{20}$ Holton, J., An Introduction to Dynamic Meteorology, Academic Press, New York, 1992.

${ }^{21}$ Wallace, J., and Hobbs, P., Atmospheric Science, Academic Press, New York, 1977.

${ }^{22}$ Sarpkaya, T., "Decay of Wake Vortices of Large Aircraft," AIAA Journal, Vol. 36, 1998, pp. 1671-1679. 